\title{
Strengthening mental health systems in low- and middle-income countries: recommendations from the Emerald programme
}

Maya Semrau, Atalay Alem, Jose L. Ayuso-Mateos, Dan Chisholm, Oye Gureje, Charlotte Hanlon, Mark Jordans, Fred Kigozi, Crick Lund, Inge Petersen, Rahul Shidhaye and Graham Thornicroft

\section{Background}

There is a large treatment gap for mental, neurological or substance use (MNS) disorders. The 'Emerging mental health systems in low- and middle-income countries (LMICS)' (Emerald) research programme attempted to identify strategies to work towards reducing this gap through the strengthening of mental health systems.

\section{Aims}

To provide a set of proposed recommendations for mental health system strengthening in LMICs.

\section{Method}

The Emerald programme was implemented in six LMICs in Africa and Asia (Ethiopia, India, Nepal, Nigeria, South Africa and Uganda) over a 5-year period (2012-2017), and aimed to improve mental health outcomes in the six countries by building capacity and generating evidence to enhance health system strengthening.

\section{Results}

The proposed recommendations align closely with the World Health Organization's key health system strengthening 'building blocks' of governance, financing, human resource development, service provision and information systems; knowledge transfer is included as an additional cross-cutting component. Specific recommendations are made in the paper for each of these building blocks based on the body of data that were collected and analysed during Emerald.

\section{Conclusions}

These recommendations are relevant not only to the six countries in which their evidential basis was generated, but to other LMICs as well; they may also be generalisable to other non-communicable diseases beyond MNS disorders.

\section{Declaration of interest}

None.

\section{Keywords}

Global mental health; health systems; health system strengthening; healthcare delivery.

\section{Copyright and usage}

(C) The Royal College of Psychiatrists 2019. This is an Open Access article, distributed under the terms of the Creative Commons Attribution-NonCommercial-NoDerivatives licence (http://creativecommons.org/licenses/by-nc-nd/4.0/), which permits noncommercial re-use, distribution, and reproduction in any medium, provided the original work is unaltered and is properly cited. The written permission of Cambridge University Press must be obtained for commercial re-use or in order to create a derivative work.
This paper proposes strategies for the strengthening of mental health systems in low- and middle-income countries (LMICs) that have arisen out of the work of the 'Emerging mental health systems in LMICs' (Emerald) research programme. The 5-year programme (2012-2017) aimed to improve mental health outcomes in six LMICs in Africa and Asia (Ethiopia, India, Nepal, Nigeria, South Africa and Uganda) by building capacity and generating evidence to enhance health system strengthening. ${ }^{1,2}$ The research programme thereby worked towards improving mental healthcare in LMICs, and ultimately contributing to a reduction in the mental health treatment gap. This gap means that currently a large percentage of people with mental, neurological or substance use (MNS) disorders (generally around 75\% in LMICs) do not receive any form of treatment or care for their MNS disorder, and an even greater number (up to $95 \%$ in LMICs) do not receive minimally adequate treatment. $^{3-6}$

\section{Method}

Emerald entailed the coordination of several components, which aligned closely with the World Health Organization's (WHO's) 'building blocks' for health system strengthening, ${ }^{7}$ i.e. (a) governance; (b) mental health financing; (c) human resources for mental health system development; (d) service provision; and (e) mental health information systems; additionally, knowledge transfer was included as a cross-cutting component. Recommendations from each of these components are outlined below. These are based on the body of data that were collected and analysed during Emerald, and which are presented in detail in the papers of this thematic series $^{2,8-13}$ and in other publications cited throughout this paper. The recommendations may be applicable beyond the six countries in which the evidence for them were collected, as well as to noncommunicable diseases other than MNS disorders.

\section{Results}

A summary of our recommendations is provided in Appendix 1. In addition, some practical pointers are listed in Appendix 2.

\section{Governance}

Poor governance was identified as an important barrier to effective integration of mental healthcare within primary healthcare settings. To address this, the Emerald programme identified the following key governance strategies (see Petersen et al, Abdulmalik et al, Hanlon et al, Marais \& Petersen, Mugisha et al, and Upadhaya et $a l^{14-19}$ for further details): strengthening capacity of managers at subnational levels and/or policymakers at the national level to develop and implement integrated plans; strengthening key aspects of the essential health system building blocks to promote 
responsiveness, efficiency and effectiveness; developing workable mechanisms for intersectoral collaboration, as well as community and patient engagement; and developing innovative approaches to improve mental health literacy and achieve stigma reduction. A recurring challenge for good governance is adequate financing, which can be addressed via evidence-based advocacy and budgetary re-prioritisation.

\section{Mental health financing}

The Emerald programme showed that as part of effective planning, countries can benefit from undertaking an assessment of the human and financial resources needed to scale-up a package of evidencebased care and prevention strategies for priority MNS disorders. Emerald developed, tested and applied a new module for the OneHealth tool (see http://www.avenirhealth.org/software-onehealth.php), to estimate the costs and health impact of mental health service scale-up in the six Emerald countries. ${ }^{20}$ The findings were that costs of scale-up of key mental health services are modest in absolute terms (under US $\$ 0.50$ per head of population for psychosis, depression and epilepsy in Ethiopia, Nepal and Uganda), but with substantial anticipated improvements in health. However, these costs are still considerably in excess of current allocations because of low prioritisation to mental health.

Across the six diverse Emerald LMICs, a relatively consistent picture emerged - that there is limited financial risk protection for households affected by MNS disorders. Emerald's findings indicated that households living with an MNS disorder constitute a key vulnerable population, who have a high risk of chronic poverty and intergenerational poverty transmission, and who therefore require development assistance. Development efforts can target the 'upstream' determinants of mental health: violence, poverty, inadequate housing, unemployment, lack of basic amenities, poor education, experience of trauma and stigma; which can significantly reduce the risk of MNS disorders among those not yet affected and increase the ability to cope with financial hardships as a result of MNS disorders among those already affected. This could be implemented in the form of financial assistance such as disability grants or cash transfers for people living with an MNS disorder. It is also essential to improve access to mental healthcare, preferably delivered through primary care and community-based healthcare platforms, for example by including mental healthcare within universal health coverage plans.

In order to improve access to services and move towards universal health coverage for people with MNS disorders, greater financial protection needs to be given to individuals and households with MNS disorders, preferably through explicit inclusion of MNS disorders in ongoing national insurance schemes or programmes (see the paper by Chisholm et al in this thematic series ${ }^{9}$ ). Universal or social health insurance models offer the most promising avenues to achieve sustained resourcing for mental health, as well as improving the efficiency with which resources are used for mental health, by moving away from specialised care to primary healthcare services. There is a need for continued advocacy for mental health services to be included in the benefits packages under these social health insurance financing models, to increase the political will and tackle the low priority given to mental health.

\section{Human resources}

In regard to human resources for mental health system development, the key findings from Emerald show that it is essential to expand access to integrated mental healthcare. ${ }^{21}$ For this to happen, task-sharing of mental healthcare through training of general health workers needs to be augmented by interventions to empower and equip mental health patients and their caregivers.
This should be supplemented with capacity-building of service planners/managers and in-country researchers in LMICs in mental health system strengthening. ${ }^{22-26}$ It is important for these capacity-building activities to be carefully planned and implemented, taking contextual variations into account; for this, situational analyses or needs assessments should be conducted in order to inform the capacity-building activities. ${ }^{23,27-29}$ Governments and donors can facilitate human resource development by making involvement of patients/caregivers a requirement and by providing resources for capacity-building. It is also important to ensure that service managers and planners are equipped with good knowledge about mental health system strengthening and understand the importance of integration of mental health into primary healthcare, so that they can influence the thinking of frontline health workers in primary care settings and provide the necessary support for any integration efforts. Care providers in health institutions need to be equipped with good knowledge about mental health system strengthening too, to ensure that the actual actors in the care provision are included and to facilitate effective and efficient implementation.

Emerald developed models of best practice for training activities and research collaborations between the high-income countries (HICs) and LMICs. The programme highlighted the importance of appropriateness, reciprocity and sustainability within these collaborations, and that people in LMICs should drive the process of, and be equal partners in, any training activities. When funding multicountry research programmes, embedding $\mathrm{PhD}$ fellowships for LMIC researchers is an investment in the next generation of researchers from the global South. Emerald supported ten $\mathrm{PhD}$ students and two MSc students, who now have the potential to make important contributions to mental health systems research in LMICs through their PhD/MSc work and through their future activities. Furthermore, evaluation of capacity-building efforts is imperative but requires dedicated resources (see paper by Evans-Lacko et $a l^{8}$ in this thematic series on how capacity-building efforts were evaluated within Emerald; see also Hanlon $e t a l^{30}$ ).

\section{Service provision}

Although great strides have been made in the development of policy and legislative frameworks that support integrated care, the Emerald programme found that the implementation of these mental health policies and plans remains a challenge, requiring technical support, such as manuals, standard operating procedures, 'Train the Trainer' technical support, and monitoring and evaluation using continuous quality improvement to embed integration.

The Emerald programme also found that the integration of mental health into primary healthcare (PHC) requires more than just technical (continued) training and structured supervision of healthcare providers in the required clinical skills (for example through the Mental Health Gap Action Programme (mhGAP)). This training needs to be accompanied by systems strengthening of all the basic building blocks of the PHC system to support integrated mental healthcare. This includes systems interventions to support integrated person-centred collaborative continuing care of chronic and multimorbid conditions at an organisational level. lt also refers to the need for workforce preparedness interventions that include relational leadership skills, clinical communication skills and emotional coping skills (see paper in this thematic series by Petersen et $a l^{10}$ ). A clinical communication skills training module that promotes a person-centred approach was developed as part of Emerald and introduced into the national scale-up efforts in South Africa to support integrated mental healthcare into primary care services. It has also been adapted for use in Ethiopia as part 
of the adaptation of integrated chronic care guidelines called the Practical Approach to Care Kit (PACK). ${ }^{31}$

Systems strengthening interventions to support integrated mental healthcare varied across the six Emerald countries depending on country needs but were found to improve patients' experience of overall chronic care across the countries. Furthermore, Emerald found that strengthening of the community platform is also important to promote intersectoral collaboration, mental health literacy, reduce stigma and empower patients and caregivers for support and self-care.

\section{Mental health information systems}

In order to guide the process of scaling up of mental healthcare, a revised health management information system (HMIS) that includes mental health indicators is needed in LMICs. ${ }^{32,33}$ Through a broad-based consensus building process that included a cross-country Delphi study and consultative workshops, ${ }^{34}$ Emerald developed a set of indicators that can be used within the routine mental health information systems in LMICs to monitor the provision of mental health services in PHC (see Jordans et al's paper ${ }^{11}$ in this thematic series for the list of indicators and the evaluation of use of the indicators). With a limited set of indicators, Emerald aimed to assess 'effective coverage' including financial coverage, both of which are needed for the assessment of implementable universal health coverage.

The set of indicators, after a brief training of health workers, were introduced in practice. Emerald assessed its performance and perceived utility, finding mostly high and increasing levels of completeness and accuracy of data completion, even though some indicators fell behind in perceived utility. The results showed that it is feasible, useful and acceptable to use the indicators for routine monitoring of mental healthcare within existing HMIS in LMICs (see papers by Jordans et $a l^{11}$ and Ahuja et $a l^{12}$ in this thematic series for further details). We therefore propose these indicators to be considered for incorporation into existing health information systems, and adopted within the WHO mhGAP implementation strategy.

\section{Knowledge transfer}

This has been included here as a cross-cutting component, which although not one of the WHO's 'building blocks' for health system strengthening - we consider to be an important requirement within health system research. It is imperative that research evidence is communicated effectively and efficiently to a wide range of stakeholders, including those who may apply this information in practice to improve treatment and care. Knowledge transfer from research into policies and patient care could be accelerated by involving patients and carers. For effective communication of research results, there is a need for multiple dissemination strategies. This may include meetings with stakeholders such as advocacy meetings or community groups, websites, social media, leaflets, newsletters, policy briefs, research papers, conferences, annual reports, videos and press conferences and releases. The paper by Ayuso-Mateos et $\mathrm{al}^{13}$ in this thematic series goes into further details on this, as well as the impact that the knowledge transfer efforts within Emerald had on mental health service delivery and policy planning within the six Emerald countries.

\section{Discussion}

The Emerald programme created a rich body of evidence to inform proposed strategies for strengthening mental health systems in LMICs. This evidence was collected in six LMICs in Africa and
Asia, but has applicability beyond those countries to other LMICs and potentially to underresourced areas in $\mathrm{HICs},{ }^{35}$ as well as to non-communicable diseases other than MNS disorders. Indeed, some of the tools developed during Emerald have already been successfully used in Zimbabwe (see Hendler et al and Kidia et $a^{36,37}$ ), and we would encourage other countries to follow suit. The evidence collected during Emerald has resulted in a set of recommendations, which we hope will be useful in informing models of best practice not just in the six Emerald countries but also in other LMICs and possibly other HICs worldwide on how to enhance health systems so that services for people with MNS disorders are improved.

Maya Semrau, Research Fellow in Implementation Research, Centre for Global Health Research, Brighton and Sussex Medical School; and Centre for Global Mental Health, Institute of Psychiatry, Psychology and Neuroscience, King's College London, UK; Atalay Alem, Professor, Department of Psychiatry, School of Medicine, College of Health Sciences, Addis Ababa University, Ethiopia; Jose L. Ayuso-Mateos, Chairman and Director, Department of Psychiatry, School of Medicine, Universidad Autónoma de Madrid; and Hospital Universitario de La Princesa, Instituto de Investigación Sanitaria Princesa (IIS Princesa), Centro Investigación Biomédica en Red de Salud Mental (CIBERSAM), Spain; Dan Chisholm, Programme Manager for Mental Health, Regional Office for Europe, Department of Mental Health and Substance Abuse, World Health Organization, Switzerland; Oye Gureje, Professor of Psychiatry and Director, WHO Collaborating Centre for Research and Training in Mental Health, Neurosciences and Substance Abuse, Department of Psychiatry, University of Ibadan, Nigeria; and Professor Extraordinary, Department of Psychiatry, Stellenbosch University, South Africa; Charlotte Hanlon, Reader in Global Mental Health, Centre for Global Mental Health, Institute of Psychiatry, Psychology and Neuroscience, King's College London, UK; and Adjunct Associate Professor, Department of Psychiatry, School of Medicine, College of Health Sciences, Addis Ababa University, Ethiopia; Mark Jordans (ID, Reader, Centre for Global Mental Health, Institute of Psychiatry, Psychology and Neuroscience, King's College London, UK: Fred Kigozi, Senior Consultant Psychiatrist and Researcher, Butabika National Referral and Teaching Hospital, Uganda; Crick Lund (iD), Professor of Public Mental Health, Alan J Flisher Centre for Public Mental Health, Department of

Psychiatry and Mental Health, University of Cape Town, South Africa; and Professor of Global Mental Health and Development, Centre for Global Mental Health, Health Service and Population Research Department, Institute of Psychiatry, Psychology and

Neuroscience, King's College London, UK; Inge Petersen, Research Professor and Director, Centre for Rural Health, School of Nursing and Public Health, University of KwaZulu-Natal, South Africa; Rahul Shidhaye, Clinical Psychiatrist, Public Health Foundation of India; and CAPHRI School for Public Health and Primary Care, Maastricht University, the Netherlands; Graham Thornicroft, Professor of Community Psychiatry, Centre for Global Mental Health and Centre for Implementation Science, Institute of Psychiatry, Psychology and Psychiatry, King's College London, UK

Correspondence: Maya Semrau, Centre for Global Health Research, Brighton and Sussex Medical School, University of Sussex, Falmer, Brighton BN1 9PS, UK. Email: M.Semrau@bsms.ac.uk

First received 28 Aug 2018, final revision 31 Nov 2018, accepted 2 Dec 2018

\section{Funding}

The research leading to these results was funded by the European Union's Seventh Framework Programme (FP7/2007-2013) under grant agreement $n^{\circ}$ 305968. The funder had no role in study design, data collection and analysis, decision to publish or preparation of the manuscript. G.T. is supported by the National Institute for Health Research (NIHR) Collaboration for Leadership in Applied Health Research and Care (CLAHRC) South London and by the NIHR Applied Research Centre (ARC) at King's College London NHS Foundation Trust, and the NIHR Applied Research and the NIHR Asset Global Health Unit award. The views expressed are those of the author(s) and not necessarily those of the NHS, the NIHR or the Department of Health and Social Care. G. $T$. receives support from the National Institute of Mental Health of the National Institutes of Health under award number R01MH100470 (Cobalt study). G T. is Supported by the Partnership (MR/ R023697/1) awards. M.S. is supported by the NIHR Global Health Research Unit for Neglected Tropical Diseases at the Brighton and Sussex Medical School. D.C. is a staff member of the World Health Organization. The authors alone are responsible for the views expressed in this publication and they do not necessarily represent the decisions, policy or views of the World Health Organization. G.T., C.H., C.L., A.A. and I.P. are funded by the NIHR Global Health Research Unit on Health System Strengthening in Sub-Saharan Africa, King's College London (GIRU $16 / 136 / 54)$ using UK aid from the UK Gor tion are those of the authors and not necessarily those of the NIHR or the Department of Health and Social Care. C.H. additionally receives support from AMARI as part of the DELTAS Africa Initiative (DEL-15-01)

\section{Acknowledgements}

The partner organisations involved in Emerald were Addis Ababa University (AAU), Ethiopia; Butabika National Mental Hospital (BNH), Uganda; ARTTIC, Germany; HealthNet TPO, Netherlands; King's College London (KCL), UK; Public Health Foundation of India (PHFI), India; Transcultural Psychosocial Organization Nepal (TPO Nepal), Nepal; Universidad Autonoma de Madrid (UAM), Spain; University of Cape Town (UCT), South Africa; University of Ibadan (UI), Nigeria: University of KwaZulu-Natal (UKZN), South Africa; and World Health Organization 
group consisted of A.A. (AAU), J.L.A.-M. (UAM), D.C. (WHO), Dr Stefanie Fülöp (ARTTIC), O.G. (UI), C.H. (AAU), M.J. (TPO Nepal; KCL), F.K. (BNH), C.L. (UCT), I.P. (UKZN), R.S. (PHFI) and G.T. (KCL) Parts of the programme were also coordinated by Ms Shalini Ahuja (PHFI), Dr Jibril Omuya Abdulmalik (UI), Ms Kelly Davies (KCL), Ms Sumaiyah Docrat (UCT), Dr Catherine Egbe (UKZN), Dr Sara Evans-Lacko $(\mathrm{KCL})$, Dr Margaret Heslin $(\mathrm{KCL})$, Dr Dorothy Kizza $(\mathrm{BNH})$, Dr Lola Kola (UI), Dr Heidi Lempp (KCL), Dr Pilar López (UAM), Ms Debra Marais (UKZN), Ms Blanca Mellor (UAM), Mr Durgadas Menon (PHFI), Dr James Mugisha (BNH), Ms Sharmishtha Nanda (PHFI), Dr Anita Patel (KCL), Ms Shoba Raja (BasicNeeds, India; KCL), Dr Maya Semrau (KCL), Mr Joshua Ssebunya (BNH), Mr Yomi Taiwo (UI), and Mr Nawaraj Upadhaya (TPO Nepal). The Emerald programme's scientific advisory board included A/Professor Susan Cleary (UCT), Erofessor Derege (University of Melbourne, Australia), Mr Patrick Onyango (TPO Uganda), Professor Jose Luis Salvador Carulla (University of Sydney, Australia), and Dr R. Thara (Schizophrenia Research Foundation (SCARF), India). The following individuals were members of the Emerald consortium: Dr Kazeem Adebayo (UI), Ms Jennifer Agha (KCL), Ms Ainali Aikaterini (WHO), Dr Gunilla Backman (London School of Hygiene and Tropical Medicine; KCL), Mr Piet Barnard (UCT), Dr Harriet Birabwa (BNH), Ms Erica Breuer (UCT), Mr Shveta Budhraja (PHFI), Amit Chaturvedi (PHFI), Mr Daniel Chekol (AAU), Mr Naadir Daniels (UCT), Mr Bishwa Dunghana (TPO Nepal), Ms Gillian Hanslo (UCT), Ms Edith Kasinga (BNH), Ms Tasneem Kathree (UKZN), Mr Suraj Koirala (TPO Nepal), Professor Ivan Komproe (HealthNet TPO), Dr Mirja Koschorke (KCL), Mr Domenico Lalli (European Commission), Mr Nagendra Luitel (TPO Nepal), Dr David McDaid (KCL), Ms Immaculate Nantongo (BNH), Dr Sheila Ndyanabangi (BNH), Dr Bibilola Oladeji (UI), Professor Vikram Patel $(\mathrm{KCL})$, Ms Louise Pratt $(\mathrm{KCL})$, Professor Martin Prince $(\mathrm{KCL})$, MS M. Miret (UAM), Ms Warda Sablay (UCT), Mr Bunmi Salako (UI), Dr Tatiana Taylor Salisbury (KCL), Dr Shekhar Saxena (WHO), Ms One Selohilwe (UKZN), Dr Ursula Stangel (GABO:mi), Professor Mark Tomlinson (UCT), Dr Abebaw Fekadu (AAU) and Ms Elaine Webb (KCL).

\section{Appendix 1}

\section{Summary of recommendations}

(a) Governance: poor governance needs to be addressed as a key barrier to the effective integration of mental healthcare.

(b) Mental health financing: the mental health module of the OneHealth tool is useful to estimate the human and financial resources needed to scale-up a package of evidence-based care and prevention strategies for priority mental, neurological or substance use (MNS) disorders. Emerald's findings show that it is essential to improve access to services and move towards universal health coverage for people with MNS disorders; for this, greater financial protection needs to be given to individuals and households living with MNS disorders, preferably through explicit inclusion of MNS disorders in ongoing national insurance schemes or programmes, in particular social health insurance models and targeted poverty alleviation programmes.

(c) Human resources: there needs to be capacity-building of mental health patients, caregivers, service planners/managers and researchers in low- and middle-income countries (LMICs) in mental health system strengthening. Training activities and collaborations should be carefully planned, implemented and evaluated, that emphasise appropriateness, reciprocity, sustainability and equality in partnerships; governments and/or donors need to make resources available for this.

(d) Service provision: the scale-up of integrated mental healthcare into primary healthcare (PHC) in LMICs is far more complex than adding packages of care to existing PHC services. Leveraging existing health system processes that are synergistic with chronic care are important, as well as initiatives to strengthen some of the basic building blocks of the healthcare system to create a more enabling platform for integrated mental healthcare. Furthermore, community strengthening is important to promote empowering of patients and caregivers for support and self-care; and the implementation of mental health policies and plans requires technical support.

(e) Mental health information systems: Emerald developed and evaluated a set of indicators (measuring treatment need, utilisation, quality and costs) that can be used within routine mental health information systems in LMICs to monitor the effective coverage of mental health services in PHC. These indicators should be incorporated into existing health information systems, and adopted within the WHO Mental Health Gap Action Programme implementation strategy.

(f) Knowledge transfer: research evidence should be communicated effectively and efficiently to a wide range of stakeholders, including those who may apply this information in practice to improve treatment and care, using a wide array of platforms suitable for the target audience.

\section{Appendix 2}

\section{Practical pointers for mental health system strengthening in low- and middle-income countries}

(a) Moving towards universal health coverage for people with MNS disorders requires consideration of the resources needed to scale-up services and also consideration of the fair and sustainable mechanisms for providing enhanced financial protection to affected households.

(b) Ensure that there is a strong focus on capacity-building of patients, policymakers, planners and researchers to support mental health system strengthening.

(c) Any country that is envisioning the integration of mental health into primary healthcare should review the requirements and processes across the health system building blocks. This paper provides guidance around this process.

(d) Ensure that routine health information systems include mental health indicators so that mental healthcare needs and services can be routinely monitored.

\section{References}

1 Semrau M, Evans-Lacko S, Alem A, Ayuso-Mateos JL, Chisholm D, Gureje O, et al. Strengthening mental health systems in low- and middle-income countries: the Emerald programme. BMC Med 2015; 13: 79.

2 Thornicroft G, Semrau M. Health system strengthening for mental health in lowand middle-income countries: introduction to the Emerald programme. BJPsych Open 2019; this issue.

3 Alonso J, Liu Z, Evans-Lacko S, Sadikova E, Sampson N, Chatterji S, et al. Treatment gap for anxiety disorders is global: results of the World Mental Health Surveys in 21 countries. Depress Anxiety 2018; 35: 195-208.

4 Degenhardt L, Glantz M, Evans-Lacko S, Sadikova E, Sampson N, Thornicroft G, et al. Estimating treatment coverage for people with substance use disorders: an analysis of data from the World Mental Health Surveys. World Psychiatry 2017: 16: 299-307.

5 Thornicroft G, Chatterji S, Evans-Lacko S, Gruber M, Sampson N, AguilarGaxiola S, et al. Undertreatment of people with major depressive disorder in 21 countries. Br J Psychiatry 2017; 210: 119-24.

6 Wang PS, Aguilar-Gaxiola S, Alonso J, Angermeyer MC, Borges G, Bromet EJ, et al. Use of mental health services for anxiety, mood, and substance disorders in 17 countries in the WHO world mental health surveys. Lancet 2007; 370: 841-50.

7 World Health Organization. Everybody's Business: Strengthening Health Systems to Improve Health Outcomes - WHO's Framework for Action. WHO, 2007.

8 Evans-Lacko S, Hanlon C, Alem A, Ayuso-Mateos JL, Chisholm C, Gureje O, et al. Evaluation of capacity-building strategies for mental health system strengthening in low- and middle-income countries for service users and caregivers, policymakers and planners, and researchers. BJPsych Open 2019; this issue.

9 Chisholm D, Docrat S, Abdulmalik J, Alem A, Gureje O, Gurung D, et al. Mental health financing challenges, opportunities and strategies in low- and middleincome countries: findings from the Emerald project. BJPSych Open 2019; this issue.

10 Petersen I, van Rensburg A, Kigozi F, Semrau M, Hanlon C, Abdulmalik J, et al. Scaling up integrated mental health in six low- and middle-income countries: obstacles, synergies and implications for systems reform. BJPsych Open 2019; this issue.

11 Jordans M, Chisholm D, Semrau M, Gurung D, Abdulmalik J, Ahuja S, et al. Evaluation of performance and perceived utility of mental healthcare indicators in routine health information systems in five low- and middle-income countries. BJPsych Open 2019; this issue. 
12 Ahuja S, Hanlon C, Chisholm D, Semrau M, Gurung D, Abdulmalik J, et al. Experience of implementing new mental health indicators within information systems in six low- and middle-income countries: qualitative study. BJPsych Open 2019; this issue.

13 Ayuso-Mateos JL, Miret M, Lopez-Garcia P, Alem A, Chisholm D, Gureje O, et al. Effective methods for knowledge transfer to strengthen mental health systems in low- and middle-income countries. BJPsych Open 2019; this issue.

14 Petersen I, Marais D, Abdulmalik J, Ahuja S, Alem A, Chisholm D, et al Strengthening mental health system governance in six low- and middle-income countries in Africa and South Asia: challenges, needs and potential strategies. Health Policy Plann 2017; 32: 699-709.

15 Abdulmalik J, Kola L, Gureje O. Mental health system governance in Nigeria: challenges, opportunities and strategies for improvement. Global Ment Health 2016; 3: e9.

16 Hanlon C, Eshetu T, Alemayehu D, Fekadu A, Semrau M, Thornicroft G, et al. Health system governance to support scale up of mental health care in Ethiopia: a qualitative study. Int J Ment Health Syst 2017; 11: 38

17 Marais DL, Petersen I. Health system governance to support integrated mental health care in South Africa: challenges and opportunties. Int J Ment Health Sys 2015; 9: 14

18 Mugisha J, Ssebunnya J, Kigozi FN. Towards understanding governance issues in integration of mental health into primary health care in Uganda. Int J Ment Health Syst 2016; 10: 25.

19 Upadhaya N, Jordans MJD, Pokhrel R, Gurung D, Adhikari RP, Petersen I, et al. Current situations and future directions for mental health system governance in Nepal: findings from a qualitative study. Int J Ment Health Syst 2017; 11: 37.

20 Chisholm D, Heslin M, Docrat S, Nanda S, Shidhaye R, Upadhaya N, et al. Scaling-up services for psychosis, depression and epilepsy in sub-Saharan Africa and South Asia: development and application of a mental health systems planning tool (OneHealth). Epidemiol Psychiatr Sci 2016; 19: 1-11.

21 Thornicroft G, Ahuja S, Barber S, Chisholm D, Collins PY, Docrat S, et al. Integrated care for people with long-term mental and physical conditions in low- and middle-income countries: narrative review. Lancet Psychiatry 2019; 6: $174-86$.

22 Keynejad R, Semrau M, Toynbee M, Evans-Lacko S, Lund C, Gureje O, et al Building the capacity of policy-makers and planners to strengthen menta health systems in low- and middle-income countries: a systematic review. BMC Health Serv Res 2016; 16: 601.

23 Lempp $\mathrm{H}$, Abayneh S, Gurung D, Kola L, Abdulmalik J, Evans-Lacko S, et al. Service user and caregiver involvement in mental health system strengthening in low and middle income countries: a cross-country qualitative study. Epidemiol Psychiatr Sci 2018; 27: 29-39.

24 Semrau M, Alem A, Abdulmalik J, Docrat S, Evans-Lacko S, Gureje O, et al. Developing capacity-building activities for mental health system strengthening in low- and middle-income countries for service users and caregivers, service planners and researchers. Epidemiol Psychiatr Sci 2018; 27: 11-21.
25 Semrau M, Lempp H, Keynejad R، Evans-Lacko S, Mugisha J, Raja S, et al. Service user and caregiver involvement in mental health system strengthening in lowand middle-income countries: systematic review. BMC Health Serv Res 2016; 16: 79 .

26 Thornicroft G, Semrau M. Mental health capacity building in low and middle income countries: the Emerald Programme. Epidemiol Psychiatr Serv 2018; 27: $1-2$.

27 Abayneh S, Lempp H, Alem A, Alemayehu D, Eshetu T, Lund C, et al. Service user involvement in mental health system strengthening in a rural African setting: qualitative study. BMC Psychiatry 2017; 17: 187.

28 Gurung D, Upadhaya N, Magar J, et al. Service user and care giver involvement in mental health system strengthening in Nepal: a qualitative study on barriers and facilitating factors. Int J Ment Health Syst 2017; 11: 30.

29 Samudre S, Shidhaye R, Ahuja S, et al. Service user involvement for mental health system strengthening in India: a qualitative study. BMC Psychiatry 2016; 16: 269.

30 Hanlon C, Semrau M, Alem A, Abayneh S, Abdulmalik J, Docrat S, et al. Evaluating capacity-building for mental health system strengthening in lowand middle-income countries for service users and caregivers, service planners and researchers. Epidemiol Psychiatr Sci 2018; 27: 3-10.

31 Fairall L, Bateman E, Cornick R, Faris G, Timmerman V, Folb N, et al. Innovating to improve primary care in less developed countries: towards a global model. BMJ Innov 2015: 1: 196-203.

32 Upadhaya N, Jordans MJD, Abdulmalik J, Ahuja S, Alem A, Hanlon C, et al. Information systems for mental health in six low- and middle-income countries: cross country situation analysis. Int J Ment Health Syst 2016; 10: 60.

33 Ahuja S, Shidhaye R, Semrau M, Thornicroft G, Jordans M. Mental health information systems in resource-challenged countries: experiences from India. $B J$ Psych Int 2018; 15: 43-6.

34 Jordans MJD, Chisholm D, Semrau M, Upadhaya N, Abdulmalik J, Ahuja S, et al. Indicators for routine monitoring of effective mental healthcare coverage in lowand middle-income settings: a Delphi study. Health Policy Plann 2016; 31: 1100-6.

35 Bhugra D, Pathare S, Joshi R, Kalra G, Torales J, Ventriglio A. A review of mental health policies from Commonwealth countries. Int J Soc Psychiatry 2018; 64: 3-8.

36 Hendler R, Kidia K, Machando D, Crooks M, Mangezi W, Abas M, et al. 'We are not really marketing mental health': Mental health advocacy in Zimbabwe. PLOS One 2016; 11: e0161860.

37 Kidia K, Machando D, Mangezi W, Hendler R, Crooks M, Abas M, et al. Mental Health in Zimbabwe: a health systems analysis. Lancet Psychiatry 2017; 4: 876-86. 